\title{
Recent development of experimental techniques for high-pressure mineral physics under simulated mantle conditions
}

\author{
H. J. MUELLER*, F. R. SCHILLING, C. LATHE and J. LAUTERJUNG \\ GFZ Potsdam in der Helmholtz-Gemeinschaft, Telegraphenberg, D-14473 Potsdam, Germany
}

(Received 1 August 2006; revised 31 August 2006; in final form 4 October 2006)

\begin{abstract}
The Earth's mantle has a mass of about $4.08 \times 10^{21}$ ton and represents $68 \%$ of the total mass of the Earth. The Earth's mantle is only accessible by indirect methods, such as seismological studies. The interpretation of seismic data from the Earth's deep interior requires measurements of the physical properties of Earth materials under experimental simulated mantle conditions. MAX80, maximum conditions of about $12 \mathrm{GPa} / 2000 \mathrm{~K}$, and the sister apparatus MAX200x, designed to reach $25 \mathrm{GPa}$ and $2400 \mathrm{~K}$, are installed at HASYLAB beamlines. Both apparatus are equipped for XRD with a Ge-solid-state detector, for transient ultrasonic interferometry, as well as with a radiography system to measure the change of volume and shape of the sample under in situ conditions.

Some recent results on the elastic wave velocities at the non-quenchable high- $P$-low- $P$ clinoenstatite transition and on some innovative experiments to improve the potentials of multi-anvil apparatus in terms of maximum pressure are presented.
\end{abstract}

Keywords: Multi-anvil apparatus; DIA; Opposed-anvil third stage; Ultrasonic interferometry; X-ray diffraction; X-radiography

\section{Introduction}

Direct access to Earth's deep interior by drilling is limited to about $15 \mathrm{~km}$, mostly because of the temperature limit for the drilling mud acting as driving medium for the downhole-motors, coolant and transport medium for the cuttings. Beyond this horizon, only geology itself can support us. At special geological locations, rocks from 30 to $40 \mathrm{~km}$ depths are exhumed and can be sampled at the surface. Recently even diamond bearing rocks from a depth of 150-300 km were found. But it is an open question how far these samples really represent huge parts of the upper mantle. Both exhumation and eruption of breccia require very special states of the surrounding mantle. Any data from greater depths are based on indirect observations, mostly the velocity, diffraction and reflexion of seismic waves excited from earthquakes and cruising through the whole Earth at high $P$ and $T$. The interpretation of those data require high-pressure experiments under in situ conditions, i.e. experiments under experimentally

*Corresponding author. Email: hans-joachim.mueller@gfz-potsdam.de

High Pressure Research

ISSN 0895-7959 print/ISSN 1477-2299 online @ 2006 Taylor \& Francis

http://www.tandf.co.uk/journals

DOI: $10.1080 / 08957950601092606$ 
simulated mantle conditions. The high intense X-rays produced in up-to-date synchrotrons have an extraordinary meaning for these experiments, because the simultaneous measurement of elastic and structural data under in situ conditions is crucial for the material interpretation of seismic data from great depths.

Contrary to classical experimental petrology that investigates high-pressure run products after the experiment is terminated by quenching and using the metastability, modern mineral physics performs the experiments in real time under in situ conditions. It is also able to investigate non-quenchable mineral phases and has also direct access to reaction kinetics. The combination of three different methods - seismology, ultrasonic interferometry and X-ray phase analysis - gives a powerful virtual access to Earth's deep interior.

Figure 1 shows the most probable pressure and temperature distribution inside the Earth as well as the limits of the different high-pressure devices available for in situ experiments today. From the five systems reaching mantle conditions, in principle belt and piston-cylinder are ruled out because the sample is more or less inaccessible for a synchrotron beam. Three options remain - toroid-cell (Paris-Edinburgh-cell), multi-anvil apparatus and diamond anvil cell (DAC) (figure 1). The toroid-cell can be used for a big specimen and has a $360^{\circ}$ free radiation access and, therefore, it is very important for experiments with neutrons. But the pressure is too low for mantle research. The DAC is just the opposite case. It can reach even core conditions up to $300 \mathrm{GPa}$, but the specimen is very small $\left(10^{-11}-10^{-14} \mathrm{~m}^{3}\right)$ resulting in high temperature gradients and secondary stress from laser heating. Unfortunately, this is a strong limitation for process monitoring on multi-component systems as far as grain boundary

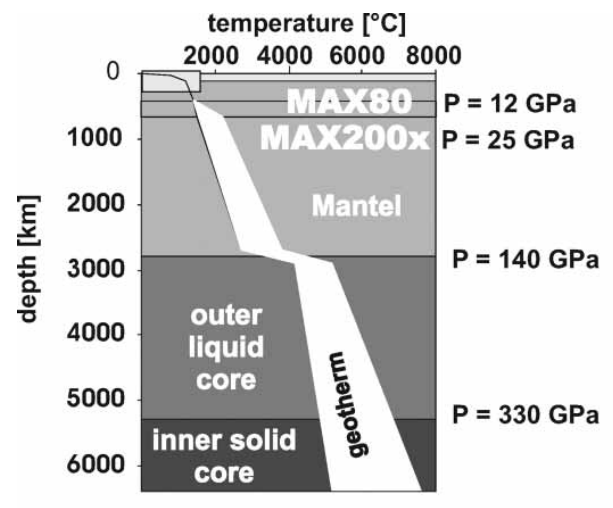

a

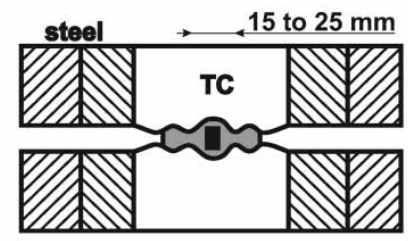

C

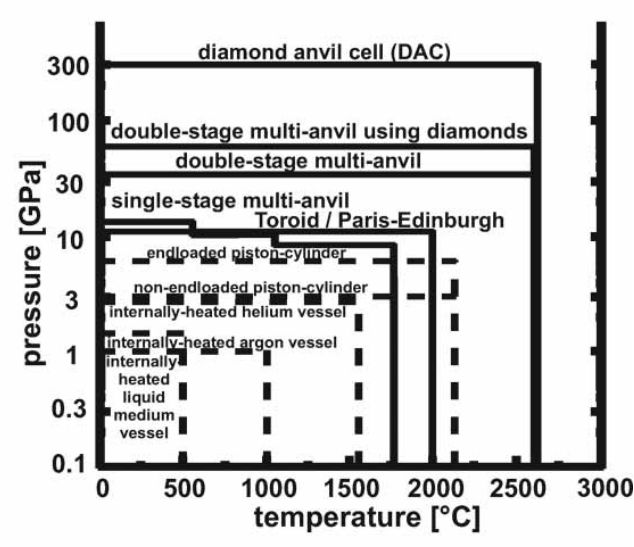

b 
reactions and scaling are essential. In principle, toroid-cell and DAC compress the sample volume uni-axial. A hydrostatic or pseudo-hydrostatic pressure is produced by the shape and internal friction of the mineral pressure transmitting medium (toroid-cell) and the metal sealing as well as the liquid or gaseous pressure medium in the DAC [1]. Contrary to this, multi-anvil devices [5-7] are based on the three-axial compression of the sample volume and the extensive lateral anvil support. The specimen dimensions can be equal in all three directions of space. There are no principle limits for pressure, temperature and sample volume. The usual sample volume is $10^{3}$ to $10^{6}$-fold bigger than in DAC, but the maximum pressure is still limited to some tens of GPa. Further discussions are focussed on multi-anvil devices established at synchrotron beamlines.

\section{Multi-anvil techniques}

\subsection{Single-stage multi-anvil DIA}

A DIA system is composed of six anvils moving uniformly and rectangularly to each other toward the sample centre (see figure 1e). The set-up for the experiment consists of a boron epoxy cube containing the sample itself, the heater, the pressure standard, the thermocouple, some insulation sleeves, buffer rods, reflectors etc. [2,8]. Boron epoxy is selected as pressure transmitting medium because of its hardness, low X-ray absorption and small scattering cross section. Increasing load on the cube results in its plastic deformation. A portion of the cube material is squeezed out into the gap between the anvils forming the gaskets preventing the cube material from further squeezing.

\subsection{Double-stage multi-anvil DIA}

Depending on anvil material and truncation, single-stage multi-anvils are restricted to maximum pressures of 8-12 GPa, representing a depth level of 100-150 km in the Earth. To make transition zone - the interface between upper and lower Earth's mantle at a depth between about 440 and $660 \mathrm{~km}$ - conditions accessible, double-stage multi-anvil systems are needed. Double-stage means, an additional set of eight anvils which are put between the first-stage anvils and the set-up. These anvils are truncated resulting in a octahedral space in the centre of the configuration. Using tungsten carbide (TC) for the second-stage anvils, peak pressures of more than $30 \mathrm{GPa}$ are possible; using sintered diamond anvils, even more than $70 \mathrm{GPa}$ [9-12]. The double-stage systems reach higher pressures because the lateral anvil support is stronger, tensile stress is cut back at the interfaces and the material can be optimized - outside tougher, inside harder. To get higher pressures, the octahedron is made from harder materials, e.g. MgO. The internal set-up is very similar to that of a single-stage device. The gaskets are not formed from the set-up, but are insets made from pyrophyllite [13]. There is another version of doublestage multi-anvils, called Kawai-Walker-module. The eight-anvil configuration is compressed between two loadframes having tapering cavities for the second-stage configuration. This version is simpler to use, but is more limited in peak pressures and X-ray access.

\subsection{Approach to higher pressures in multi-anvil DIAs}

Early multi-anvil experiments using a tetrahedral configuration were carried out by Hall [14]. Deduced from experiments with leakproof sealed split-sphere configurations driven by an oil pressure vessel, Kawai et al. [15] published the dependence of maximum pressure on 
the number of anvils, i.e., the more the sample approaches spherical shape, the higher the pressure. Our first approach aimed to check if this is also valid for single-stage multi-anvil devices. Six TC-anvils of 4-mm truncation got 1.5-mm deep pyramidal cavities at the front face. The corresponding set-up containing a mixture of $\mathrm{NaCl}$ and $\mathrm{BN}$ as pressure standard had a tetrakis hexahedral shape, i.e. it was a body having 24 isosceles triangular plane surfaces of the same shape (figure $2 a$ and $b$ ). The anvils were equipped with prefabricated gaskets [16] for an increased lateral support. The pressure was measured by XRD using the Decker scale [17]. However, no essential increase of the peak pressure was observed (figure $2 \mathrm{f}$ ). Contrary to the Kawai [15] experiment with 20 multi-pistons (anvils), the 24 planes of our experiment were not loaded with anvils acting independently, i.e. moving uniformly to the centre of the set-up. Consequently, the resulting plane compressing the set-up was still the original square-shaped frontface of the anvils.

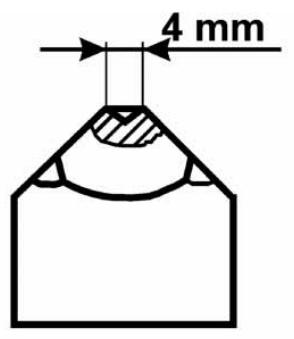

a

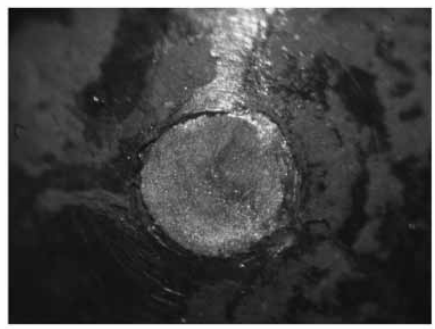

b
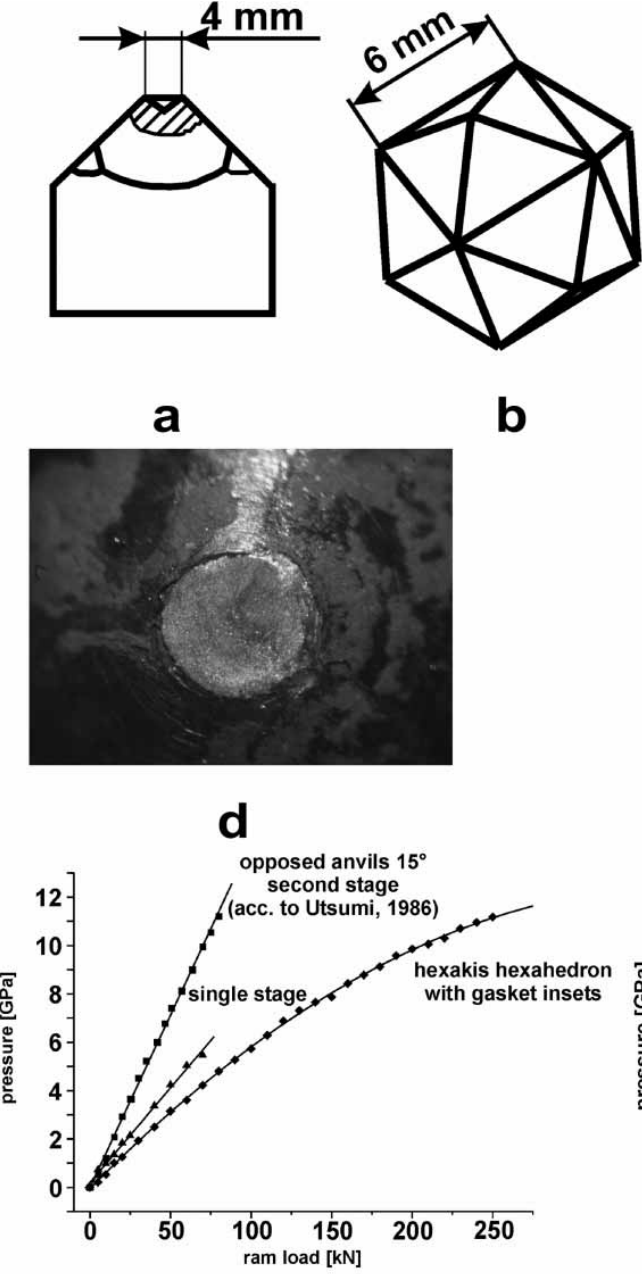

e

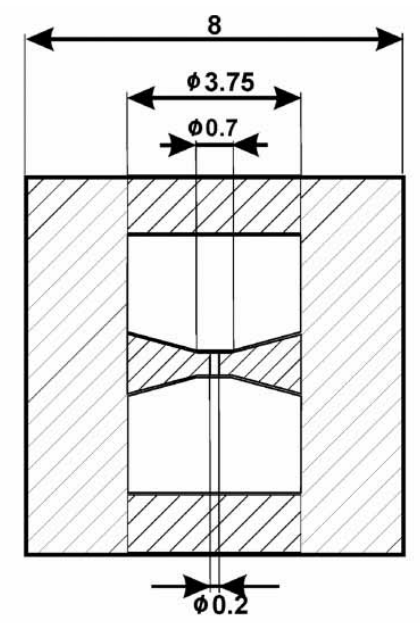

C

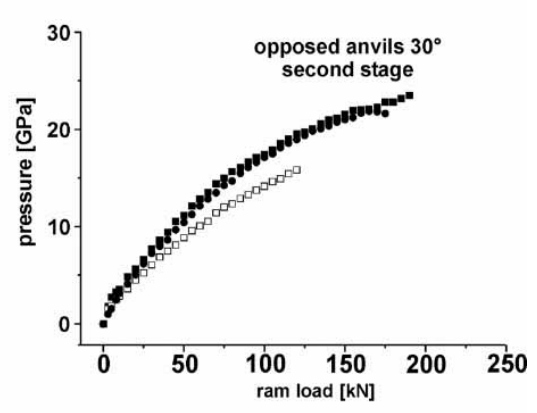

f

Figure 2. Mult-anvil pressure improvement, (a) Single-stage anvil modified for hexakis hexahedron set-up; (b) Hexakis hexahedron loaded with a $\mathrm{NaCl} / \mathrm{BN}$ sample for pressure measurement; (c) Opposed-anvil set-up for single-stage multi-anvils (according to ref. [26]); (d) Front face of an opposed anvil after the run (see figure 2c); (e) Pressure measurement for the $15^{\circ}$-opposed anvil set-up (see design $2 \mathrm{c}$ ) and the hexakis hexahedron configuration in comparison to the original 8-mm cubic set-up; (f) Pressure measurement for $30^{\circ}$-opposed anvil set-ups. 
Utsumi et al. [18] published a technique to reach $60 \mathrm{GPa}$ by using sintered diamond anvils as second stage in a single-stage DIA-type multi-anvil apparatus. We modified the set-up and used fine-grained tungsten carbide as the anvil material inside an 8-mm boron epoxy cube. Both opposed anvils had a conical shape with a cylindrical shaft. As specimen, we used the same 1:1 mixture by volume of $\mathrm{NaCl}$ and $\mathrm{BN}$, as used in the first experiment. The sample was enclosed in a matrix made from boron epoxy with an internal diameter of $0.2 \mathrm{~mm}$. In comparison to the original 8-mm cell assembly, the maximum pressure was doubled (figure $2 \mathrm{c}$ and $\mathrm{f}$ ). Because the pressure was lower than published by Utsumi et al. [18] and Wang and Utsumi [19], even though the post-experimental examination which we performed did not show any plastic deformation of the TC-anvils (see figure 2d) indicating exceeding the maximum strength of the material, we modified the set-up. The angle of the cone was increased from $15^{\circ}$ to $30^{\circ}$. The specimen was replaced by cast resin containing 5 vol.\% Au-powder with a grain size of $1.5-3 \mu \mathrm{m}$. Both changes aimed to increase the pressure efficiency by increasing the load on the sample being pore-free to the greatest possible extent. The pressure measured by XRD using the Anderson scale [20] reached $25 \mathrm{GPa}$, i.e. more than the 4-fold pressure as was reached by the original single-stage set-up (figure $2 \mathrm{f}$ ). This pressure corresponds to the results for usual double-stage multi-anvils using a much more complicated eight-cube second stage set-up equipped with small truncations of about $3 \mathrm{~mm}$ and using presses with a load of about 1500 tons or more.

\subsection{Triple-stage multi-anvil DIA}

The set-up successfully used in the single-stage DIA MAX80 installed at a bending magnet beamline at DESY-HASYLAB was modified for experiments in the new double-stage DIA MAX200x installed at the hard wiggler HARWI-II beamline at DESY-HASYLAB. The top and bottom part of an $\mathrm{MgO}$ octahedron with $10 \mathrm{~mm}$ edge length was removed by two parallel cuts. A second octahedron of the same size was cut at positions shorter by twice the saw blade thickness to be able to reconstruct an original undeformed octahedron. After loading the central hole with the opposed anvil set-up, the octahedron was completed by cementing both caps on the rear side of the anvils and the central $\mathrm{MgO}$ part, respectively. This way we reached nearly $50 \mathrm{GPa}$ in a triple-stage configuration (figure 3). Broken third-stage anvils in some experiments indicate that this is at the pressure limit for TC. But even with broken opposed
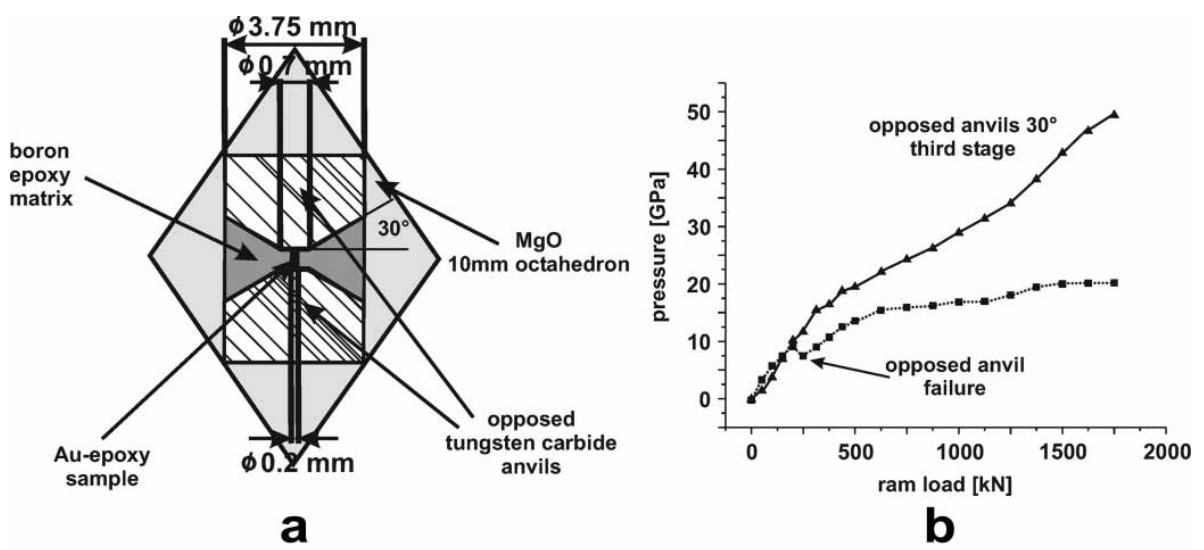

Figure 3. (a) Opposed anvil set-up $\left(30^{\circ}\right)$ as third stage in multi-anvil device; (b) Pressure measurement for triple-stage multi-anvil experiments. 
anvils, the pressure was about $10 \%$ higher than in the original double-stage configuration, because the second stage becomes less deformable by replacing essential $\mathrm{MgO}$-volume parts by TC-parts.

\section{Measurements under in situ conditions}

\subsection{X-ray diffraction $(X R D)$}

$\mathrm{X}$-ray diffraction under in situ conditions is the primary aim for establishing multi-anvil devices at a synchrotron. Energy-dispersive XRD uses the high flux of synchrotron radiation for the observation of reaction kinetics, because saving of a diffraction pattern takes about a minute. The synchrotron beam of a diameter of 50-100 $\mu \mathrm{m}$ is guided between the anvils through the gaskets. Two high-precision slits are adjusted in a way that only X-rays diffracted at the sample in the center of the set-up hit the Ge-solid state detector. The remaining undiffracted beam is absorbed in a beam stop.

For angle-dispersive XRD, a double-crystal, fixed-offset monochromator with silicon (3 11 1) single-crystals is used. The monochromator is calibrated in the wavelength range between 0.4 and 0.6. A CCD-detector with a diameter of $133 \mathrm{~mm}$ detects the four detection fan cut out from the diffraction rings by the four gaps between the TC-anvils. Angle-dispersive XRD has a higher resolution for structure refinements under in situ conditions. However, this requires much longer exposition times, because only a little part of the X-ray spectrum is used.

\subsection{Pressure measurement}

In multi-anvil devices established at synchrotrons, pressure is measured by XRD of the pressure standard by evaluating the deformation of the unit cell of cubic crystals, e.g. $\mathrm{NaCl}, \mathrm{Au}, \mathrm{Pt}$, $\mathrm{MgO}$, which are located as close as possible to the sample or mixed with the sample in case of a crushed specimen, e.g. [17,20,21]. Only the photon flux of synchrotron radiation is sufficient to perform this measurement in real time. It is an indirect method which refers to the equation of state $(\mathrm{EoS})$ for the used pressure standard. If synchrotron radiation is not available, a pressure calibration by observing the change of the electrical resistivity at a phase transition is another option. After several experiments resulting in one point each, a calibration curve can be constructed. The drawback is the much lower accuracy because of the limited pressure reproducibility of multi-anvil experiments, and that the pressure cannot be measured during any experiment this way. For ultrasonic experiments, a further, very elegant way exists. If the velocities of elastic compressional and shear waves are measured simultaneously, the compressibility and, consequently, the pressure can be calculated standard-free and exactly inside the sample $[16,22]$. However, the method requires expensive calculations, i.e. using current PC-technology, the pressure data are not available in time during the experiment.

\subsection{Temperature generation and measurement}

The experimental simulation of Earth's deep interior conditions require not only the generation of pressure, but also of temperature. In multi-anvil devices, this is made by electrical resistance heating. Because of its deformability and machinability, low X-ray absorption as well as low resistivity graphite is the preferred material for the tube-shaped heater contacted from two of the TC-anvils. Because graphite transforms to diamond at elevated pressures and temperatures, heaters are also made from $\mathrm{LaCrO}_{3}$, various carbides or rhenium sheet. For pure 
XRD-experiments, a phase-shifting control is a good solution. Unfortunately, it produces a lot of electrical noise. Consequently, for ultrasonic interferometry or high frequency electrical measurements, dc- or pure sinusoidal hf-power supply is needed.

The sample temperature is measured by a thermocouple. The connecting wires are brought out through the anvil gaps. For precise measurements, one has to take into account corrections for the pressure dependence of the thermoelectric effect [23]. Note: In the near future, a complete new option for temperature measurements at high-pressure conditions will be available based on the Johnson-noise, i.e. the evaluation of voltage noise in a resistor being proportional to the thermodynamical temperature [24]. The test experiments are in progress. When available, this technique may have an extraordinary impact on up-to-date mineral physics because this is also an absolute method like the already existing ultrasonic method for pressure measurement.

\subsection{Ultrasonic interferometry - data transfer function}

Corresponding to the unique relevance of seismology for geophysics, elastic wave velocity measurements under in situ conditions are crucial. Because of sample size and design of the high-pressure devices, the way to do this is ultrasonic interferometry, i.e. the evaluation of signals reflected from the polished front and rear faces of the sample. Ultrasonic waves are generated and detected using the piezoelectric effect. Because of high conversion factor and temperature stability, $\mathrm{LiNbO}_{3}$ is the prefered material for high-pressure mineral physics. The classical sweep technique sends all sinusoidal tone bursts of the pass band one after another to the sample and saves the response of the system. The technique is very time consuming. Therefore, the data transfer function technique (DTF) was developed $[13,23,25]$. The transducer is excited by a raised-cosine pulse resulting in emittance of the whole frequency band at the same time. The response of the working section to this excitation is the DTF comprising all monochromatic responses inside the frequency pass band. To reproduce these responses for each single frequency, the DTF is convolved with the single frequencies. The technique is very fast. It requires just a few seconds to save the DTF, together with energydispersive XRD performed at a synchrotron that gives the power for time resolved (transient) measurements and makes unquenchable phase transitions accessible for high-pressure mineral physics. Ultrasonic measurements with double-stage multi-anvils require triple-mode ultrasonic transducers generating compressional and shear waves at the same time because there is only space for attaching one transducer to the second-stage anvils at the outward truncation. Figure $4 \mathrm{a}$ and $\mathrm{c}$ show the ultrasonic set-up before the run and some results measured with sweep- and DTF-technique for the unquenchable high-pressure clinoenstatite $\left(\mathrm{MgSiO}_{3}\right.$, HCEn)-low-pressure clinoenstatite (LCEn) transition at high pressure, high-temperature conditions performed in conjunction with in situ XRD $[13,16]$.

\subsection{X-radiography}

Ultrasonic interferometry requires an exact knowledge of the sample length under in situ conditions, because the measured interference patterns depend not only on elastic wave velocity but also on sample length. For X-radiography, the blades of the primary slits are opened so far that the synchrotron beam has a slightly higher diameter than the sample length. After penetrating the high-pressure cell, the resulting X-ray shadow graph is partially converted by fluorescence of a 0.1-mm thick Ce:YAG-crystal (by courtesy of IKZ) into a visible image. An aluminum-coated mirror decouples the optical from the non-converted X-ray image. The optical image is magnified by a microscope and captured by a CCD-camera. The sample length 


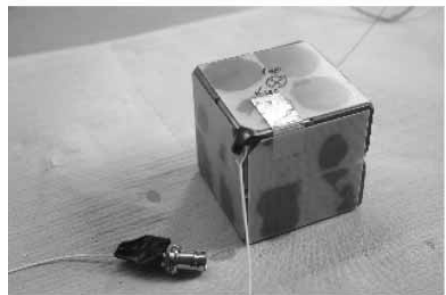

a

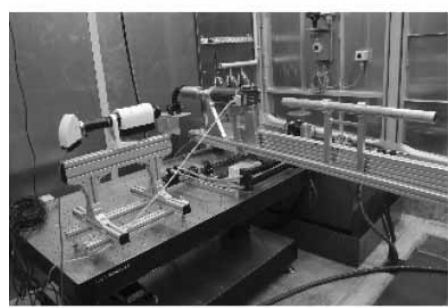

C

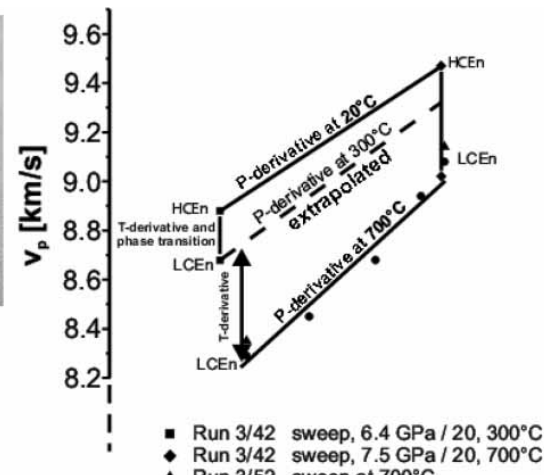

- Run $3 / 52$ sweep at $700^{\circ} \mathrm{C}$

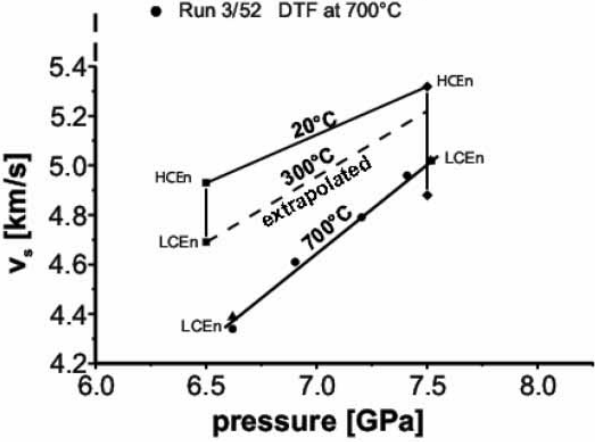

b

Figure 4. Ultrasonic measurements in conjunction with synchrotron radiation (in situ XRD), (a) Ultrasonic set-up with triple-mode transducer for double-stage multi-anvil device MAX200x; (b) Elasic wave velocities $v_{\mathrm{p}}$ and $v_{\mathrm{s}}$ measured with sweep and DTF-technique at the unquenchable high pressure clinoenstatite $\left(\mathrm{MgSiO}_{3}\right.$, HCEn) - low-pressure clinoenstatite (LCEn) transition at high pressure, high-temperature conditions in conjunction with in situ XRD; (c) Equipment for energy-dispersive XRD and X-radiography at multi-anvil device MAX200x.

measurement is performed by image processing [13]. Figure 4 shows the $\mathrm{X}$-radiography equipment of the double-stage multi-anvil MAX200x together with goniometer, slits and Ge-solid state detector for energy-dispersive XRD.

\section{Summary and perspective}

High-pressure mineral physics under in situ conditions is crucial for the interpretation of geophysical data from Earth's deep interior. Understanding of the system Earth, minimizing the impact of natural catastropies to the human society and safeguarding its future by supplying resources requires more and more the understanding of Earth's mantle dynamics, i.e. the analysis of the driving forces. The availability of third generation synchrotrons, X-ray lasers and neutron spallation sources together with new high-pressure materials, e.g. single-crystal chemical vapour deposed (CVD) diamonds, binder-free polycrystalline nano particle (NPD) diamonds and achievements of information technology enable the design of new high-pressure tools and corresponding measurement techniques. High-pressure mineral physics appeared as a key discipline for recent and future geo-, planetary and material sciences.

\section{References}

[1] H.J. Mueller, F.R. Schilling and J. Lauterjung, Z. geol. Wiss 3049 (2002a).

[2] H.J. Mueller, J. Lauterjung, F.R. Schilling et al., Eur. J. Mineral. 14581 (2002b). 
[3] Mao and Hemley, 1998.

[4] Sherman and Stadtmuller, 1987.

[5] T. Yagi, Eos Trans. AGU 6918 (1988).

[6] T. Yagi, M. Akaogi, O. Shimomura et al., J. Geophys. Res. 926207 (1987a).

[7] T. Yagi, M. Akaogi, O. Shimomura et al., in High-Pressure Research in Mineral Physics, edited by M.H. Manghnani and Y. Syono (AGU, Washington, D.C., 1987b), p. 141; Geophys. Monogr. Ser. 39.

[8] H.J. Mueller, F.R. Schilling, J. Lauterjung et al., Eur. J. Mineral. 15865 (2003).

[9] T. Irifune, H. Naka, T. Sanehira et al., Phys. Chem. Min. 29645 (2002).

[10] T. Irifune, A. Kurio, S. Sakamoto et al., Nature 421599 (2003).

[11] T. Irifune, Kurio, S. Sakamoto et al., Phys. Earth Planet. Int. 143593 (2004).

[12] T. Kondo, H. Sawamoto, A. Yoneda et al., PAGEOPH 141 601(1993).

[13] H.J. Mueller, F.R. Schilling, and C. Lathe, Advance in High Pressure Mineralogy (2006), in press.

[14] H.T. Hall, Rev. Sci. Instrum. 29267 (1958).

[15] N. Kawai, S. Endoh and S. Sakata, Proc. Japan. Acad. 42626 (1966).

[16] H.J. Mueller, F.R. Schilling, C. Lathe et al., in Advances in High Pressure Technology for Geophysical Application, edited by J. Chen, Y. Wang, T. Duffy et al., chapter 21 (Elsevier B.V., 2005), p. 427.

[17] D.L. Decker, J. Appl. Phys. 423239 (1971).

[18] W. Utsumi, T. Yagi, K. Leinenweber et al., in High-Pressure Research: Application to Earth and Planetary Sciences, edited by Y. Syono and M.H. Manghnani (Terrapub., 1992), p. 37.

[19] Y. Wang and W. Utsumi, paper presented at COMPRES Annual Meeting 2005, Mohonk Mountain House, New Paltz, June 16-19 (2005).

[20] O.L. Anderson, D.G. Isaak and S.Yamamoto, J. Appl. Phys. 64(4) 1534 (1989).

[21] I. Jackson and H. Niesler, in High-Pressure Research in Geophysics, edited by S. Akimoto and M.H. Manghnani (Center for Academic Publications Japan/Tokio, 1982), p. 93.

[22] B. Li, J. Kung, T. Uchida et al., in Advances in High Pressure Technology for Geophysical Application, edited by J. Chen, Y. Wang, T. Duffy et al., chapter 3 (Elsevier B.V., 2005), p. 49.

[23] B. Li, K. Chen, J. Kung et al., J. Phys. Condens. Matter 1411337 (2002).

[24] I.C. Getting, paper presented at COMPRES Annual Meeting 2005, Mohonk Mountain House, New Paltz, June 16-19 (2005).

[25] H.J. Mueller, C. Lathe and F.R. Schilling, in Advances in High Pressure Technology for Geophysical Application, edited by J. Chen, Y. Wang, T. Duffy et al., chapter 4 (Elsevier B.V., 2005), p. 67.

[26] Utsumi, 1986. 